\title{
Layer Specification of Transplanted Interneurons in Developing Mouse Neocortex
}

\author{
Helen Valcanis and Seong-Seng Tan \\ Howard Florey Institute, The University of Melbourne, Parkville 3010, Victoria, Australia
}

\begin{abstract}
The six-layered neocortex is composed of excitatory projection neurons and inhibitory interneurons. Recent studies have established separate embryological origins for these two cellular populations. However, it remains uncertain how interneurons arising from the subcortical ganglionic eminences are able to participate in the orderly stratification of the cortical layers. A related question concerns whether or not early and late interneuron progenitors have equivalent developmental potentials. To address these issues, we performed transplantation experiments to test the fates of early- versus late-born interneuron populations using cells labeled with a genetic marker. Our results indicate that transplanted interneurons from the medial ganglionic eminence give rise to specific layers of the neocortex in an inside-out order. To test the potency of interneurons born at different ages, heterochronic transplantations were also performed. Both early- and late-born progenitors were able to switch their fates in the new environment, and, similar to projection neurons, fate-switching was dependent on progenitor receptivity to environmental cues during their last round of cell division. Our data also demonstrate, for the first time, that interneuron-layering cues are present within the medial ganglionic eminence, suggesting that, before the commencement of long-distance tangential migration, interneurons are already specified with respect to their future layer addresses. So, although the generation of diverse neuronal phenotypes in separate locations is an effective strategy to pursue separate developmental programs, our results indicate that excitatory and inhibitory neurons share similar mechanisms for integrating sequentially born neurons from two places into a single layered structure.
\end{abstract}

Key words: interneurons; GABA; development; neocortex; layering; inhibitory

\section{Introduction}

In the developing neocortex, the fate of a neuron with regards to its layer address, or whether it has excitatory or inhibitory phenotype, is dependent on two key determinants. With cortical layering, the neuronal birthday is the determining factor in assigning a neuron to a particular layer (Angevine and Sidman, 1961; Rakic, 1974). With cellular phenotype, the birthplace of the neuron is the dominant influence: excitatory projection neurons are generated locally in the pallial ventricular zone (VZ), whereas most inhibitory interneurons are born remotely in the subpallium (Van Eden et al., 1989; DeDiego et al., 1994; Anderson et al., 1997a; Tamamaki et al., 1997).

It is unclear how these separate and independent developmental programs are linked together to produce a six-layered structure containing the appropriate balance of excitatory and inhibitory neurons. The outcome is remarkable when one considers that excitatory and inhibitory neurons, in keeping with their separate morphological and functional traits, have different developmental agendas during and after cessation of cell migration (Jones, 1990). Projection neurons follow radial pathways and can extend their axons to subcortical targets even before completing their migratory journey (Schreyer and Jones, 1982; Schwartz et al., 1991). They typically have pyramidal-shaped cell

\footnotetext{
Received Sept. 3, 2002; revised Feb. 27, 2003; accepted March 6, 2003.

This work was supported by funding from the National Health and Medical Research Council and by an equipment grant from the Helen MacPherson Smith Trust. We thank Prof. Michael Kalloniatis and Dr. Hue-Trung Truong for kind assistance in the statistical analyses of the results.

Correspondence should be addressed to Seong-Seng Tan, Brain Development Laboratory, Howard Florey Institute, The University of Melbourne, Parkville 3010, Victoria, Australia. E-mail: stan@hfi.unimelb.edu.au. Copyright $\odot 2003$ Society for Neuroscience $\quad$ 0270-6474/03/235113-10\$15.00/0
}

bodies, arborize in layer-specific patterns, and use glutamate as their neurotransmitter (Streit, 1984). Interneurons, on the other hand, are the result of long-distance tangential cell migration from the ganglionic eminence (GE) (Anderson et al., 1997a, 2002; Tamamaki et al., 1997; Lavdas et al., 1999; Wichterle et al., 2001; Nadarajah et al., 2002) and use GABA as their neurotransmitter (Fagg and Foster, 1983). Being interneurons, they have a different axo-dendritic structure with no apical dendrite; they lack dendritic spines, and their axons arborize in the vicinity of their dendritic fields (Fairen et al., 1984; Naegele and Barnstable, 1989).

Cell lineage studies have established that specification of the projection neuron phenotype occurs at the level of the progenitor, even before the onset of neurogenesis (Tan et al., 1998). Progeny from these precursors end up in one of six layers, with neurons belonging to the same layer having common cellular morphologies and afferent-efferent connections (Gilbert and Kelly, 1975; Gilbert, 1977). Thymidine birthdating studies have showed that the destination of any given cortical neuron is correlated to its "birthday": early-born neurons are destined for layers 6,5, and 4, and later-born neurons are destined for layers 3 and 2. Transplantation studies have showed that early-born projection neurons can switch their commitment from lower to upper layer fates provided that the progenitor cell completes the late part of its cell cycle in the new environment (McConnell, 1988; McConnell and Kaznowski, 1991). These studies indicate that early projection neuron precursors are multipotent and that commitment to different layer fates is sequentially activated over the temporal sequence of neurogenesis.

The following question then arises: how are interneurons, 
which are generated contemporaneously in a subcortical location, able to integrate with the "inside-out" gradient of projection neuron morphogenesis? Interneurons make up a diverse group, with at least nine morphological subtypes and even more if neuropeptides are considered (Jones and Hendry, 1986). There is no evidence that commitment of a particular interneuron subclass is linked to its birthday in the GE, and the correlation between laminar position and interneuron subphenotypes is ambiguous. In monkey sensorimotor cortex, interneurons with smaller soma sizes tend to reside in upper layers, whereas larger interneurons tend to be found in lower layers, but there is widespread overlap across different layers; in the rat, the opposite is true, with the largest interneurons distributed across layers 2 and 3 (Hendry and Jones, 1981; Lin et al., 1986). Birthdating studies that combine GABA immunocytochemistry with $\left[{ }^{3} \mathrm{H}\right]$ thymidine autoradiography have demonstrated that interneurons also follow an inside-out pattern of corticogenesis (Miller, 1986; Peduzzi, 1988). However, it is unclear whether GABA cells in a given layer have resulted from specific layer commitment before their emigration from the GE or from nonspecific mechanisms (e.g., elimination of GABA cells that have landed in inappropriate destinations). If interneurons are committed to specific layers before their migratory journey, it would raise additional questions regarding their pluripotency and layer plasticity. The purpose of the current study is to address these issues.

Transplantation experiments are able to examine whether cortical neurons are restricted or unrestricted in potential by exposing them to homologous or heterologous environments (McConnell, 1988; McConnell and Kaznowski, 1991; Frantz and McConnell, 1996; Desai and McConnell, 2000). Those studies using neocortical progenitors have revealed a heirarchy of layer potency, with early-born progenitors least restricted and late-born progenitors most restricted. Because those experiments used donor tissue from the cortical VZ, it may be assumed that the results are representative of projection neuron behavior. However, information is unavailable for interneurons, and, to address this issue, we performed isochronic and heterochronic transplantations of medial ganglionic eminence (MGE) cells, the principal source of neocortical interneurons (Wichterle et al., 2001). Our results demonstrate that layer commitment of MGE-derived interneurons occurs in a time-dependent manner, similar to projection neurons. Compared with projection neurons, our heterochronic transplants also demonstrate interneuron pluripotency of both early- and late-born cells. Together with projection neuron studies, our work highlights the complementary agendas of neurons generated in two different telencephalic compartments.

\section{Materials and Methods}

In utero cell transplantation. Isochronic and heterochronic transplantations were performed using donor tissues at embryonic day 12.5 (E12.5) and E15.5 into host embryos at both time points (day of vaginal plug detection, E0.5). Both donor and host embryos were derived from parental strains maintained on C57BL/6J $\times$ DBA hybrid backgrounds. Donor cells were sourced from a transgenic mouse line in which all neurons and glia express the lac $Z$ reporter gene (Tan and Breen, 1993). To obtain MGE cells, forebrains were dissected in ice-cold HBSS. Using watchmaker forceps, the roof of the cerebral hemisphere was excised, exposing the GE. The VZ and subventricular zone from the MGE were carefully removed, avoiding contamination from the adjacent lateral ganglionic eminence (LGE) and interventricular septum. MGE tissue from all littermates were pooled together and prepared for injection by mechanical dissociation with forceps, followed by enzymatic digestion $(10 \mathrm{~min})$ in HBSS containing $0.125 \%$ trypsin (Thermotrace, Melbourne, Australia) and $0.1 \%$ DNaseI (Roche Diagnostics, Mannheim, Germany). The tryp- sin was inactivated by addition of $10 \%$ fetal calf serum, centrifuged $(2000$ $\mathrm{rpm}$ ) into a pellet, and the cells were resuspended in HBSS with $0.1 \%$ DNaseI at a concentration of $1 \times 10^{5}$ cells/ $\mu$ l using a hemocytometer. Cells were kept at $4^{\circ} \mathrm{C}$ until they were ready for transplantation.

For isochronic transplantation, pregnant mice containing E12.5 or E15.5 embryos were anesthetized using Nembutal ( $0.6 \mathrm{mg}$ of sodium pentobarbitone per $10 \mathrm{gm}$ of body weight), and the uterine horns were exposed and gently immersed in a culture dish containing $0.25 \% \mathrm{MgSO}_{4}$ on a warm $\left(30^{\circ} \mathrm{C}\right)$ stage Under fiber optic lighting, each embryo was manipulated through the uterine wall until the position of the lateral ventricle $(\mathrm{LV})$ was discernible. Using a transplacental injection technique, a bevelled glass micropipette (tip diameter of 50-100 $\mu \mathrm{m}$ ) containing a suspension of donor cells (single cells and clumps) was introduced through a puncture hole (accessed with a 30 gauge hypodermic needle) created in the uterine wall. Cell transfer was effected using a mouth-controlled tubing attached to the pipette. Each embryo received $\sim 1 \mu$ l of cell suspension containing $1 \times 10^{5}$ cells. The procedure was repeated for every conceptus except for the one adjacent to the cervix on either side. The omission of the most proximal embryos was found to be correlated with reduced postsurgical contractions and abortions. Injections were performed using E12.5 donor cells (into E12.5 recipients; $n=64$ ) and E15.5 donor cells (into E15.5 recipients; $n=44$ ). After surgical recovery, the operated mice were returned to their cages, and the pups were reared until 6 weeks of age. For heterochronic transplantation, E12.5 cells were injected into E15.5 brains $(n=37)$. The complementary experiment was also performed, injecting E15.5 cells into E12.5 brains $(n=50)$. After E15.5, the MGE becomes difficult to distinguish from the LGE.

To label transplanted neurons undergoing the last round of cell division in the host environment, recipient embryos were exposed to a single dose of 5-bromodeoxyuridine (BrdU) administered to the peritoneal cavity of the mother $(50 \mathrm{mg} / \mathrm{kg}$ body weight $) \sim 5 \mathrm{~min}$ after cell transplantation into the last embryo. To label neurons (before transplantation) undergoing the last mitotic division in the donor MGE environment, BrdU was delivered to the mother of the donor embryos $8 \mathrm{hr}$ before cell dissection. This was to allow for sufficient time for cells that had taken up the BrdU label to complete their "S-phase" in the donor environment.

Control experiments to observe the effects of the transplantation procedure were performed using donor cells obtained from neocortical VZ (E12.5 or E13.5) transplanted into the LV of host embryos (E12.5 or E14.5).

Histology. After a lethal injection of Avertin, host animals (aged 6 weeks or more) were perfused for $10 \mathrm{~min}$ by intracardial perfusion with $4 \%$ paraformaldehyde in $0.1 \mathrm{~m}$ phosphate buffer, $\mathrm{pH} 7.2$, containing 2 $\mathrm{mm} \mathrm{MgCl}{ }_{2}$ and $5 \mathrm{~mm}$ EGTA, and the brains were removed for $10 \mathrm{~min}$ after fixation. After cryoprotection with $20 \%$ sucrose in phosphate buffer, brains were embedded in OCT (Tissue-Tek, Torrance, CA) for cryosectioning. Coronal sections $(60 \mu \mathrm{m})$ were obtained for 4-chloro-5bromo-3-indolyl- $\beta$-D-galactopyranoside (X-gal) histochemistry to visualize $\beta$-galactosidase $(\beta$ gal $)$, and $10 \mu \mathrm{m}$ coronal sections were prepared for immunohistochemistry. Sections for X-gal histochemistry were incubated overnight at $37^{\circ} \mathrm{C}$ in a solution of $0.1 \% \mathrm{X}$-gal containing $2 \mathrm{~mm}$ $\mathrm{MgCl}_{2}, 5 \mathrm{~mm}$ EGTA, $0.01 \%(\mathrm{w} / \mathrm{v})$ sodium deoxycholate, $0.02 \%(\mathrm{w} / \mathrm{v})$ Nonidet P-40, $5 \mathrm{~mm} \mathrm{~K}_{3} \mathrm{Fe}(\mathrm{CN})_{6}$, and $5 \mathrm{~mm} \mathrm{~K}_{4} \mathrm{Fe}(\mathrm{CN})_{6} \cdot 6 \mathrm{H}_{2} \mathrm{O}$ in $0.1 \mathrm{M}$ phosphate buffer. The X-gal was prepared as a $4 \%$ stock in dimethylformamide and was added to the mixture just before use. The sections were washed in phosphate buffer, mounted on 3-aminopropyltriethoxy silane (AES)-coated slides, and allowed to dry overnight. The following day, the sections were defatted in alcohols and taken through Histopure before coverslipping with Permount.

To standardize data obtained from different injected brains, coronal sections $(60 \mu \mathrm{m})$ containing $\beta$ gal-positive $(\beta g a l+)$ cells were collected from the junction of posterior one-third and anterior two-thirds of the adult cortex (position, $-1.7 \mathrm{~mm}$ posterior of bregma line) (Franklin and Paxinos, 1997). The number of $\beta$ gal + cells in a single coronal section (known as reference section) was counted in each of six brains belonging to a transplantation category. Only transplanted cells falling within the cortical area bounded by the primary motor cortex and ectorhinal cortex were quantified. To map their layer positions, $\beta$ gal + cells were allocated 
to one of 10 bins radially superimposed on the area between white matter (adjacent bin 1) and pial surface (adjacent bin 10). Numerical data from six brains in each category were tabulated as histograms to indicate their cortical depths. Adjacent coronal sections $(10 \mu \mathrm{m})$ were used for immunocytochemical analysis of interneuron phenotypes (see below).

Immunohistochemistry. For double immunofluorescence, $10 \mu \mathrm{m}$ coronal sections were mounted on AES-coated slides and dried for $2 \mathrm{hr}$ before incubating in primary antibodies. To obtain double labeling of $\beta$ gal with BrdU, GABA, calretinin, neuropeptide Y (NPY), or parvalbumin, sections were processed for immunocytochemistry using either standard two- or three-step procedures. To expose the BrdU, tissues were preincubated with $2 \mathrm{~N} \mathrm{HCl}$ at $37^{\circ} \mathrm{C}$ for 45 min before addition of the primary antibody. All primary antibodies were diluted in $0.1 \mathrm{M}$ PBS containing $0.3 \%$ Triton X-100. Incubation with primary antibody overnight was followed by $1 \mathrm{hr}$ in secondary antibodies before coverslipping with Movial. In the three-step procedure, sections were incubated for 1 $\mathrm{hr}$ in fluorescein-avidin D. Primary antibodies included a purified rabbit polyclonal antibody to $\beta$ gal (1:500 dilution; 5 Prime $\rightarrow 3$ Prime, Boulder, $\mathrm{CO})$, a mouse monoclonal antibody to $\beta$ gal (1:500 dilution; Promega, Madison, WI), a mouse monoclonal antibody to BrdU (1:40 dilution; Becton Dickinson, San Jose, CA), a rabbit antibody to GABA (1:500 to 1:1000 dilution; Sigma, St. Louis, MO), a rabbit antibody to calretinin (1:200 dilution; Swant, Bellizona, Switzerland), a rabbit antibody to NPY (1:500 dilution; Sigma), and a mouse antibody to parvalbumin (1:500 dilution; Sigma). Secondary antibodies were biotinylated goat antirabbit IgG (1:200 dilution; Vector Laboratories, Burlingame, CA), donkey anti-mouse Cy3 (1:1000 dilution; Jackson ImmunoResearch, West Grove, PA), and Alexa Fluor 594-conjugated goat anti-mouse IgG (1:500 dilution; Molecular Probes, Eugene, OR). Three-step procedures were visualized with fluorescein-avidin D (1:200 dilution; Vector Laboratories). Images were captured using an Olympus Optical (Tokyo, Japan) microscope equipped with a Spot digital camera and ImagePro Plus software (Media Cybernetics, Silver Spring, MD).

To compare the range of interneuron phenotypes generated by each class of transplantation, immunoreactive cells for GABA, parvalbumin, calretinin, and NPY were quantified from coronal sections $(10 \mu \mathrm{m})$. Depending on survival and integration of transplanted cells, the number of immunoreactive neurons that could be included for comparison was found to vary. So, although the total number of cells for each antigen was variable between sections and animals, a statistical comparison was performed between interneuron subclasses generated in the same cortical regions by different transplantation categories. The positions of transplanted cells that had also taken up BrdU were ascertained using double immunofluorescence on thin coronal sections situated in the same region. As before, the layer distribution of BrdU + cells was plotted as histograms corresponding to bins marking different levels of cortical depth. Bin locations were converted into laminar positions using counterstained sections.

\section{Results}

The introduction of neuronal progenitors into the embryonic lateral ventricle is a robust method for studying neuronal fate and potency (Brustle et al., 1995; Campbell et al., 1995; Fishell, 1995; Frantz and McConnell, 1996; Olsson et al., 1998; Desai and McConnell, 2000). These studies have demonstrated the capability of transplanted progenitor cells to incorporate into the host VZ from where they migrate into telencephalic structures and subsequently differentiate into neurons (Fishell, 1995; Frantz and McConnell, 1996; Desai and McConnell, 2000). Using this transplant model, we introduced donor MGE cells into host embryos at both early and late neurogenetic periods (Fig. $1 \mathrm{~A}$ ). Isochronic and heterochronic transplantations were performed. Of 195 transplantations, 114 pups were born, giving an average survival rate of $59 \%$. Of these, $39 \%(n=44)$ contained grafted cells, judged by the integration of $\beta$ gal + neurons in the adult forebrain. For each time point, six successful transplanted animals ( $>6$ weeks) were selected for analysis.

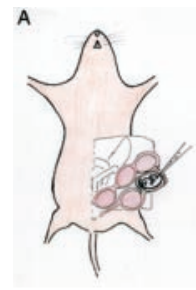

C

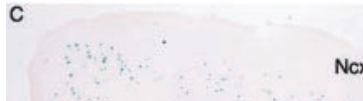

$\mathrm{Cg}$

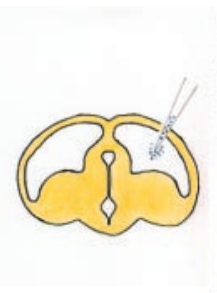

Ncx

D

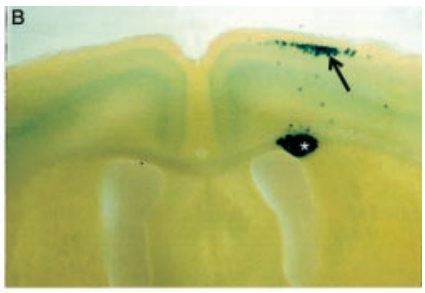

Ncx

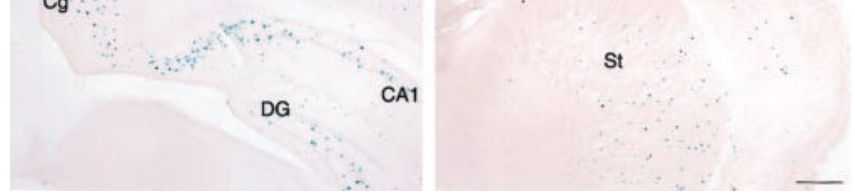

Figure 1. In utero transplantation of $\beta$ gal-labeled progenitor cells results in their incorporation into cortical and extracortical structures. $A$, Schematic diagram to illustrate introduction of cell suspensions into the developing ventricle using a transplacental approach. The loaded pipette is introduced into the ventricle, and cells are injected by pressure. $B$, Transplantation of progenitors from the neocortical VZ typically generates clumps of labeled cells (asterisk) in the white matter, with associated labeling of cortical neurons lying radially above (arrow). In this example, cells introduced into E14.5 hosts have migrated as a cluster to the upper cortical layers. $C, D$, Transplanted MGE cells do not form radial clusters; instead, they give rise to scattered cells in the neocortex (Ncx) and other structures, including cingulate cortex $(\mathrm{Cg})$, hippocampal dentate gyrus (DG) and CA fields (CA1), and striatum (St). Scale bar (in D): B, $570 \mu \mathrm{m} ; C, D, 375 \mu \mathrm{m}$.

In control experiments, we first studied the integration patterns of dissociated progenitor cells taken from the cortical VZ (E12.5 and E13.5). After injection into E12.5 and E14.5 ventricles, a small number of labeled cells have integrated successfully into the cortex, whereas other brains showed only clumps of labeled cells in white matter similar to that reported previously (McConnell, 1988; McConnell and Kaznowski, 1991; Olsson et al., 1998). Where grafted cells had migrated to the host cortex, they were often associated with a labeled clump in the white matter in radial register. In the example shown, E13.5 cells injected into E14.5 hosts had migrated to layer 2, with a few scattered cells in middle layers (Fig. $1 B$ ). The identity of the layer 2 cells were shown by immunocytochemical analysis to be glutamatergic (results not shown), suggesting that they are projection neurons. At no instance were grafted cells found in a scattered distribution, implying that the transplantation procedure per se did not initiate a scattered distribution pattern. In comparison, grafted MGE cells were never seen in radial formation but instead displayed a scattered distribution in diversed structures, including neocortex, medial and lateral limbic cortex, hippocampus (dentate gyrus and CA fields), globus pallidus, amygdala, and striatum (Fig. $1 C, D)$. Labeled MGE cells were never found in the olfactory bulb, confirming previous observations that the olfactory interneurons are derived from other striatal progenitors (Wichterle et al., 2001).

\section{Identity of transplanted MGE cells}

Neocortical interneurons are chemically diverse, and, besides GABA, they can be subdivided according to the content of their calcium-binding proteins (parvalbumin, calbindin, and calretinin) or neuropeptide (e.g., somatostatin-NPY) (Jones and Hendry, 1986; Gonchar and Burkhalter, 1997). Previous studies have reported at least $30 \%$ of all MGE cells grafted in the adult brain differentiate into GABAergic neurons (Wichterle et al., 1999). In the present study, transplanted cells in different animals $(n=3)$ 

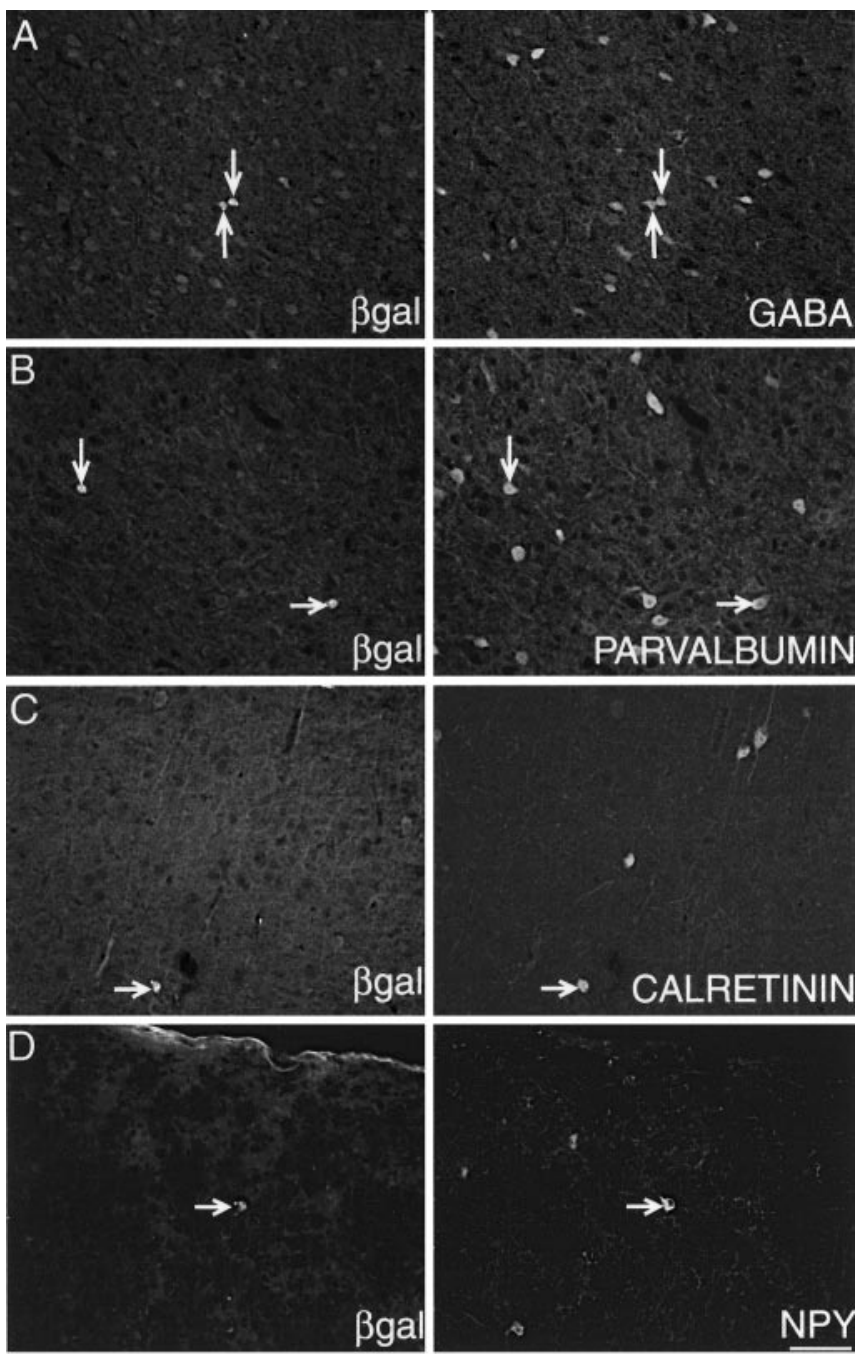

Figure 2. Transplanted MGE cells give rise to interneurons with different neurochemical subclasses (arrows). Double immunofluorescence using antibodies to $\beta \mathrm{gal}$ and neuropeptide demonstrate double labeling in transplanted cells for $\mathrm{GABA}(A)$, parvalbumin $(B)$, calretinin $(C)$, and neuropeptide $Y(D)$. Scale bar (in D), $50 \mu \mathrm{m}$.

Table 1. Percentage of transplanted cells expressing a particular chemical marker

\begin{tabular}{lcccc}
$\begin{array}{l}\text { Transplantation } \\
\text { categories }\end{array}$ & GABA & Parvalbumin & Calretinin & NPY \\
\hline E12.5 $\rightarrow$ E12.5 & $73 \pm 17.5$ & $73 \pm 10.7$ & 0 & $3.3 \pm 2.7$ \\
& $(n=120)$ & $(n=100)$ & $(n=118)$ & $(n=106)$ \\
E15.5 $\rightarrow$ E15.5 & $79 \pm 17.5$ & $77 \pm 8.4$ & $2.4 \pm 2.1$ & $4.5 \pm 3.9$ \\
& $(n=83)$ & $(n=84)$ & $(n=94)$ & $(n=105)$ \\
E12.5 $\rightarrow$ E15.5 & $73 \pm 13$ & $46 \pm 19$ & $2.2 \pm 1.4$ & $4 \pm 6.7$ \\
& $(n=157)$ & $(n=239)$ & $(n=307)$ & $(n=120)$ \\
E15.5 $\rightarrow$ E12.5 & $70 \pm 22$ & $71 \pm 22$ & $7.8 \pm 5.9$ & $6.2 \pm 2.7$ \\
& $(n=114)$ & $(n=128)$ & $(n=152)$ & $(n=98)$ \\
Average total (\# of cells) & $74 \pm 17.5$ & $67 \pm 15$ & $3.1 \pm 2.4$ & $4.5 \pm 4$ \\
& $(n=474)$ & $(n=551)$ & $(n=671)$ & $(n=429)$ \\
\hline
\end{tabular}

Data are expressed as the mean \pm SD. ANOVA was nonsignificant.

were stained for GABA, and, of 474 MGE-derived cells, an average of $74 \%$ were found to be GABAergic (Fig. 2 A, Table 1). Parallel analyses were also conducted for other neurotransmitters using material from the same animals (Table 1). In the case of parvalbumin, at least $50 \%$ of GABAergic cells normally contain this protein (Gonchar and Burkhalter, 1997). Our analysis revealed that $67 \%$ of transplanted cells contained parvalbumin

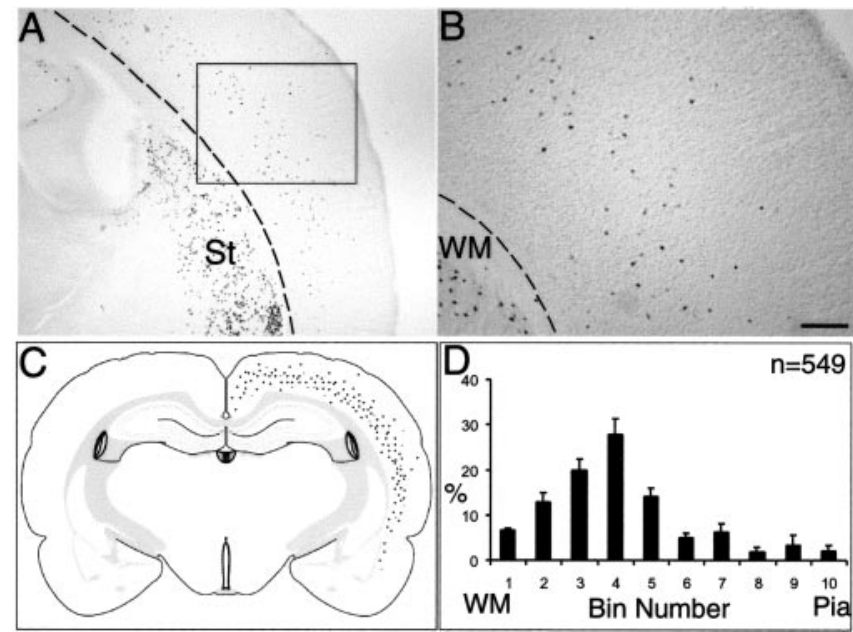

Figure 3. Isochronic transplantation of early-born (E12.5) MGE cells gave rise to mostly lower layer interneurons in the neocortex. $A, \beta \mathrm{gal}+$ cells are distributed in the lower cortical layers (boxed) and in the striatum (St). Dotted line demarcates white matter (WM). B, Higher magnification of boxed area in $A$ shows scattered cells in mostly layer 5 and occasional cells in layers 3 and 4. C, Schematic diagram showing the locations of $\beta \mathrm{gal}+$ in lower cortical layers, after combining data from the reference section of six transplanted brains (adapted from Swanson, 1998). Note that only data from neocortical cells are depicted here. D, The layer positions of $\beta \mathrm{gal}+$ cells $(n=549)$ from six different animals were assigned to 10 bins spanning from the white matter to the pia. The tabulated histograms (percentage \pm SEM) indicate lower layer positions of early-born interneurons after isochronic transplantation. Scale bar (in $B$ ): $A, 375$ $\mu \mathrm{m} ; B, 150 \mu \mathrm{m}$

(Fig. 2 B), a figure that is comparable with that reported for E13.5 transplanted MGE cells (70\%) (Wichterle et al., 2001) and for GE cells labeled with focal $\left[{ }^{3} \mathrm{H}\right]$ thymidine injections (73\%) (Anderson et al., 2002). Calretinin-positive cells were found in smaller (3\%) quantities among the grafted cells (Fig. 2C), similar to the $3 \%$ reported by Wichterle et al. (2001). In contrast, a different experimental approach using focal injections of thymidine into the ferret GE reported absence of calretinin staining among thymidine-positive cells (Anderson et al., 2002). Finally, we also examined NPY, a neuropeptide that is normally colocalized with somatostatin (Hendry et al., 1984). On average, 4\% of MGEderived cells expressed this peptide (Fig. 2D, Table 1). We also tested grafted cells with antibodies to Tbr-1, a marker for pyramidal neurons in layers 3 and 6 (Hevner et al., 2001), and GFAP, a marker for astrocytes. Neither antibody showed a positive result for the transplanted MGE cells (results not shown). In addition, we conducted immunostaining against Emx-1, a known marker for neocortical pyramidal neurons (Chan et al., 2001). These experiments also revealed a negative result for transplanted cells. The absence of MGE-derived astrocytes is in keeping with other observations that transplanted MGE cells do not normally contribute to neocortical glia but instead remain confined to the MGE vicinity as glial cells (Wichterle et al., 2001).

\section{Isochronic transplantation of MGE cells}

To examine the fate of E12.5 MGE progenitors, isochronic injections were performed using E12.5 host embryos. The results were clear, with MGE-derived cells forming a semicircle band of cells around the lower half of the neocortex (Fig. 3A), in addition to other forebrain structures mentioned before. Closer examination of the neocortical population showed that the grafted cells were diffusely scattered within the lower cortical layers (Fig. $3 A-C$ ), with a few cells distributed in the middle to upper layers (Fig. $3 B$, higher magnification). A schematic drawing, combining labeling 


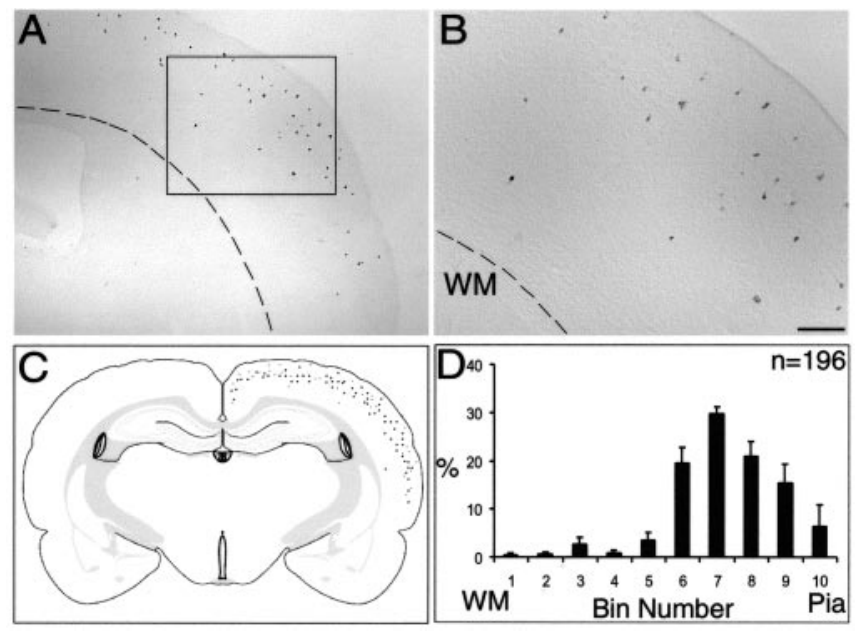

Figure 4. Isochronic transplantation of late-born (E15.5) MGE cells gave rise to mostly upper layer interneurons. $A, \beta \mathrm{gal}+$ cells are found mostly in upper layers, with hardly any cells in lower layers. Dotted line demarcates white matter (WM). B, Higher-power view of boxed area in A showing diffusely scattered cells in layers 2 and 3. C, Schematic representation of labeled cells collated from six different cortices showing upper layer fates of E15.5 isochronic transplants. $D$, The layer positions of $\beta$ gal + cells $(n=196)$ are tabulated as histograms (percentage \pm SEM), indicating that late-born cells are predominantly distributed in upper layers after isochronic transplantation. Scale bar (in $B$ ): $A, 375 \mu \mathrm{m} ; B, 150 \mu \mathrm{m}$.

patterns of reference sections from six different brains in this group, depicts the lower layer bias of transplanted E12.5 cells across the entire cortical hemisphere (Fig. $3 C$ ). To quantify the layer position of every labeled cell, the radial axis of the neocortex was divided into 10 equal bins, and every cell in the reference sections $(n=6)$ was assigned to a bin. This assessment clearly showed that a greater percentage of labeled cells $(n=549)$ were assigned to lower bins (Fig. 3D). These results would suggest that, at E12.5, MGE progenitors are committed to lower layer fates in the neocortex, in particular layer 5 (bins 3 and 4).

On the other hand, isochronic transplantation of E15.5 MGE cells gave a different picture. By far, the vast majority of labeled cells were confined to the upper part of the cortex (Fig. 4A, B). As before, cells were scattered within a narrow semicircular band situated beneath the pial surface (Fig. 4C). Quantification of labeled cells $(n=196)$ from reference sections $(n=6)$ showed that a vast majority of grafted cells had adopted upper layer fates, mostly in layers 2 and 3 but with very few cells in deeper layers (Fig. 4D).

Statistical analysis of transmitter phenotypes was conducted using the two-way ANOVA test. The results showed no difference in the range of interneuron phenotypes generated by isochronic transplantation at two different time points $(p>0.05)$.

\section{Heterochronic transplantation of MGE cells (old into young)} Previous transplantation studies of cortical projection neurons have shown that cortical VZ cells are pluripotent, but progenitors present during the early and late phases of neurogenesis have different developmental potentials (McConnell and Kaznowski, 1991; Frantz and McConnell, 1996; Desai and McConnell, 2000). To examine whether or not this was also the case for interneuron progenitors, late-born cells (E15.5) were introduced into a younger environment (E12.5) when early-born cells are known to be produced. The results show colonization of lower cortical layers by late-born cells (Fig. $5 A, B$ ), a destination normally not seen for these cells in E15.5 isochronic injections (Fig. 4). Indeed a comparison of schematic drawings with E12.5 isochronic injec-

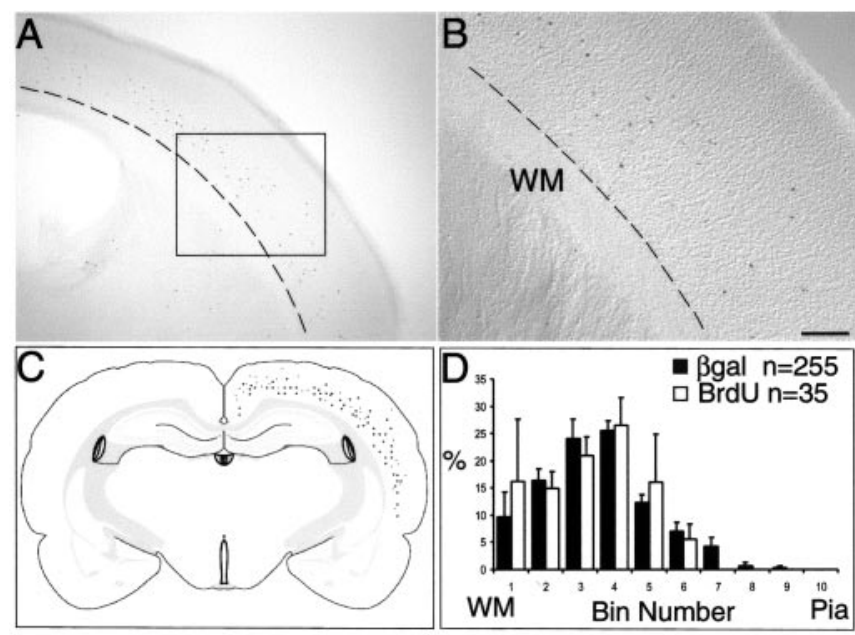

Figure 5. Late-born (E15.5) MGE cells are pluripotent and can adopt lower layer fates after heterochronic transplantation into younger embryos (E12.5). A, Transplanted late-born (E15.5) MGE cells into a younger environment (E12.5) results in fate switching into layer destinations of early-born cells. Occasionally, labeling is also seen in the striatum. Dotted line demarcates white matter (WM). B, Higher magnification of boxed area in $A$ shows the location of transplanted cells in the lower cortical layers. C, Schematic depiction of $\beta \mathrm{gal}+$ cells in the lower layers after combining data from six animals confirms lower layer positions. D, Black histograms (percentage \pm SEM) indicate the layer positions of transplanted $(n=255)$ cells that appear to have switched from upper to lower layer fates. White histograms (percentage \pm SEM) represent the layer positions of $\beta \mathrm{gal}+$ cells $(n=35)$ that also stained for BrdU, indicating a final round of cell division in the host environment. Scale bar (in $B$ ): $A, 375 \mu \mathrm{m} ; B, 150 \mu \mathrm{m}$.

tions showed almost identical distribution patterns of grafted cells (compare Fig. 5C with Fig. 3C). This conclusion was statistically verifiable, and comparison of the histograms (Fig. 3D, 5D) could not reject the null hypothesis $(p>0.05)$. Analysis of cortical depth confirmed that the majority of labeled cells are distributed in lower bins (Fig. 5D, black histograms), raising the conclusion that late-born cells have switched their layer fates and behaved like early-born neurons $(n=255)$. These results were somewhat unexpected because other studies with projection neurons have shown that late-born cortical progenitors are developmentally restricted and lacked the ability to contribute to lower layers (Frantz and McConnell, 1996).

If late-born interneurons are pluripotent, it raises the question of whether they would respond to the new environment in a cell-cycle-dependent manner. Again, studies with projection neurons have shown that transplanted progenitors are most likely to change their layer fate if they undergo a new round of cell division in the new environment (McConnell and Kaznowski, 1991). Therefore, cells that take up BrdU during S-phase DNA synthesis in the host environment are vulnerable to respecification. To mark neurons that have completed their last cell cycle in the new environment, BrdU was introduced into the host embryo after cell transplantation with the expectation that donor cells undergoing $S$-phase would take up the birthdating marker (Fig. $6 A, B)$. By using double-label immunofluorescence, transplanted cells that had undergone the last cell division in the host were detected using antibodies against $\beta$ gal (Fig. 6A) and BrdU (Fig. $6 B$, same section). Transplanted cells were found mostly in the lower layers, and, although most of them did not stain for BrdU, those that were double positive (Fig. $6 \mathrm{~A}$, cells a, d, g) were invariably located in the lower layers (Fig. 6, boxed insets with arrows). This was also confirmed by plotting the location of BrdU-labeled cells with respect to cortical depth, and the results showed that a greater percentage of $\beta$ gal $+/ \mathrm{BrdU}+$ cells were situated in the 
deeper layers (Fig. 5D, white histograms). The above results confirm that, after transplantation, progenitors that proceed to complete their final of cell division in the new environment tended to behave like early-born neurons and have settled into lower layers.

\section{Heterochronic transplantation of MGE cells (young into old)}

To study the potency of early-born MGE progenitors, E12.5 cells were injected into E15.5 ventricles. Analysis of transplanted cells $(n=376)$ showed that most cells tended to be distributed in the middle cortical layers (Fig. 7A-C), with smaller number of cells in the bins closest to the white matter and pia (Fig. 8C, black histograms). Statistical comparisons using histograms generated from isochronic E12.5 or E15.5 transplantations indicate significant differences in the pattern of distribution (ANOVA; $p<0.001$ ). After cell transplantation, the E15.5 host was injected with BrdU, resulting in uptake of neurons destined for the upper layers (Fig. $8 B$, arrows). Antibody detection for $\beta$ gal confirms midcortical layer distribution (Fig. $8 A, D)$, but immunostaining for BrdU indicates that double-labeled cells tended to be found in the upper layers (Fig. $8 D$, boxes with arrows, cells b, c). After assignment into different bins, double-positive cells (Fig. 8C, white histograms) were seen to be distributed in mostly upper layer bins (Fig. 8C). Thus, it would appear that transplanted cells that underwent a further round of mitosis in the host environment have switched from lower to upper layer fates. However, the overall result indicates a broad distribution of transplanted cells that was concentrated in the middle cortical layers, with a reduced contribution to the lower layers.

The above results raised the question of what happens to the MGE cells that were allowed to complete the last round of cell division in the donor environment before being transferred into the new host environment. Would the environmental cues encountered by these cells influence their layer destinations? To mark these cells, BrdU was injected into the donor embryo (E12.5), and the MGE was dissected after at least $8 \mathrm{hr}$ had lapsed, ensuring that some donor cells had completed S-phase in the donor environment. After injection into E15.5 hosts, the positions of double-positive cells were plotted (Fig. 9B). The results showed that cells that had undergone the last cell division in the donor MGE environment (hence retaining the BrdU marker) tended to be distributed in the middle and lower cortical layers (Fig. 9B, white histograms). Higher-power views of doublepositive cells (Fig. 9A, cells b, d, e) confirm their propensity to be distributed into middle and lower cortical layers (Fig. 9, boxed insets). Although not identical with labeling patterns of E12.5 isochronic transplants (Fig. 3), their layer locations are broadly similar. Together, these results clearly indicate that early MGE progenitors are multipotent and their layer fates can be respecified by novel environmental cues while undergoing new rounds

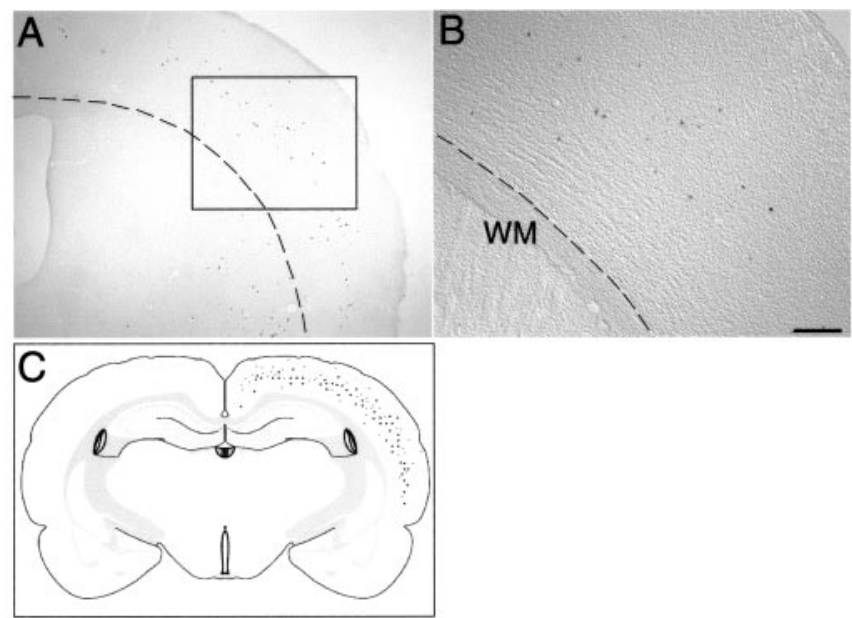

Figure 7. Heterochronic transplantation of E12.5 cells into E15.5 hosts result in layer respecification of donor cells. A, Transplanted early-born cells (E12.5) occupy principally middle cortical layers with reduced contribution to lower layers. Dotted line demarcates white matter (WM). $B$, Higher magnification of boxed area in A showing mostly middle cortical layer locations of $\beta \mathrm{gal}+$ cells. C, Schematic representation combining data from six reference sections showing the distribution of transplanted cells across a broad range of cortical layers. Scale bar (in $B$ ): $A$, $375 \mu \mathrm{m} ; B, 150 \mu \mathrm{m}$. 

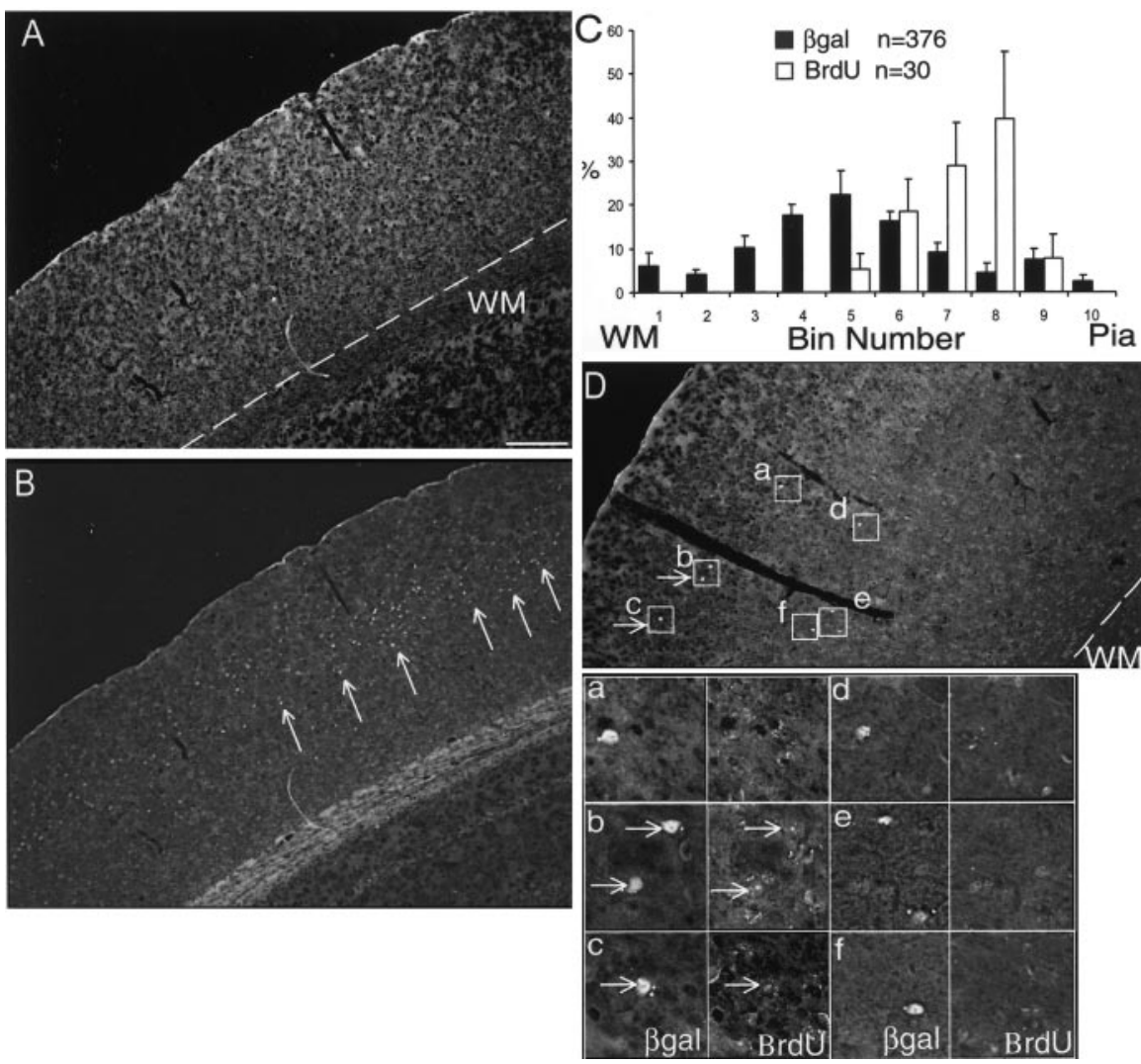

Figure 8. Heterochronic transplantation of E12.5 cells into E15.5 hosts showing cells that have undergone the last mitotic division in the older environment have adopted upper layer fates. $A, D$, Immunofluorescence for $\beta$ gal showing the location of transplanted cells (boxed) in multiple cortical layers. The presence or absence of BrdU in each of these individual cells is depicted in higher-power views at the bottom right. Arrows in $D$ point to double-labeled cells that are distributed in upper cortical layers. $B$, The same section in $A$ showing upper layer distribution for BrdU-labeled host cells after exposure to BrdU at E15.5. C, Black histograms (percentage \pm SEM) indicate the layer positions of transplanted cells that span $(n=376)$ across multiple cortical layers. White histograms (percentage \pm SEM) represent the upper layer positions of $\beta \mathrm{gal}+$ cells $(n=30)$ that are also stained for BrdU, indicating a final round of cell division in the host environment. Boxed insets show $\beta \mathrm{gal}+$ cells at higher magnification, with arrows indicating double labeling for BrdU. Scale bar (in $A$ ): $A, B, D, 150 \mu \mathrm{m}$; magnified boxes, $30 \mu \mathrm{m}$. WM, White matter.

of cell division. Conversely, progenitors that have exited from the last round of cell division while still in the younger MGE environment have retained their instructive cues and remained committed to lower layer fates.

\section{Discussion}

Isochronic transplants reveal an inside-out order of interneuron layering

Our transplantation experiments provide additional evidence that the MGE is an important source of neocortical interneurons. This adds to the general consensus, gleaned from many different types of studies, that interneurons in rodents and ferrets are derived from the underlying GE and that the neocortical neuroepithelium contributes very little, if any, cells to the inhibitory subpopulation (Marin and Rubenstein, 2001). For instance, the morphology and orientation of GABAergic neurons in the corticostriatal junction suggest tangential migration from the GE into the neocortex (Van Eden et al., 1989; DeDiego et al., 1994). Tracings of cells marked with fluorescent dyes in organotypic brain slices clearly mark the migratory pathways of cells originating from the MGE (Lavdas et al., 1999; Wichterle et al., 1999; Anderson et al., 2001; Jimenez et al., 2002; Nadarajah et al., 2002; Polleux et al., 2002). Studies with mutant mice with defective MGE development demonstrate corresponding decreases in the number of cortical interneurons (Anderson et al., 1999; Casarosa et al., 1999; Sussel et al., 1999). Focal injections, in vivo, of $\left[{ }^{3} \mathrm{H}\right]$ thymidine into the GE confirm labeling in neocortical interneurons (Anderson et al., 2002), and transplantation studies reveal the MGE, and not LGE, as the major source of invading cells into the neocortex (Wichterle et al., 2001).

Of the different investigative techniques, only cell transplantation offers the vista to distinguish between intrinsic versus extrinsic determinants of interneuron layering. In the current experiments, MGE progenitors from two separate time points of forebrain development were used as donor tissue in isochronic and heterochronic transplantations, followed by analyses of neuronal positions and phenotypes in the adult neocortex. Isochronic transplantation of early- and late-born cells were performed to address the question of whether MGE progenitors, present at early and late stages of telencephalic development, have the capacity to contribute to specific neocortical layers. The results indicate that early-born progenitors, when transplanted into similar stage hosts (E12.5), subsequently populate the lower cortical layers, principally layer 5 and, to a lesser extent, layer 6 . The small number of neurons in layer 6 may be attributable to the temporal delay caused by the crossing of transplanted cells through the ventricular lining. Conversely, late-born progenitors, when transplanted into isochronic hosts, gave rise to mainly upper layer fates (layers 2 and 3). A number of conclusions may be drawn from these observations. First, interneuron stratification in the neocortical environment occurs in an inside-out order that is linked to the age of the embryo. Together with data from neuronal birthdating studies using $\left[{ }^{3} \mathrm{H}\right]$ thymidine or BrdU (Miller, 1986; Peduzzi, 1988; Anderson et al., 2002), they indicate that the nonpyramidal neuronal sublineage obeys similar rules of cortical lamination previously defined for the pyramidal lineage, despite vastly different patterns of cell migration (Tan et al., 1998). Second, a given cohort of progenitors taken from a specific stage of MGE development appears capable of populating the entire mediolateral extent of neocortical space without any gaps, despite the lateral cortical environment being more mature compared with the medial at the time of cell transplantation (Bayer and Altman, 1991). Third, transplanted interneurons belonging to a given cohort gave rise to neurochemical sublineages that were broadly representative of the rodent neocortex (Jones and Hendry, 1986; Gonchar and Burkhalter, 1997), with GABA and parvalbumin cells greatly outnumbering calretinin and NPY. Interestingly, the ratio between different sublineages did not vary between transplanted interneurons arising from early- versus late-born progenitors. This would suggest comparable representation of different subprogenitors among early- and late-born MGE cells, or, alternatively, that the conditions giving rise to neurochemical sublineages do not appear to change over developmental time. This is in stark contrast to projection neurons in which there is a obligatory 
relationship between projection neuron phenotype (judged by morphology) and the time of its generation from the cortical neuroepithelium.

\section{Heterochronic transplants reveal MGE cell pluripotency}

Heterochronic transplantations were performed to introduce late-born progenitors into a younger environment undergoing lower layer corticogenesis and vice versa. These experiments have the potential to throw light on whether progenitors belonging to early or late cohorts are, respectively, restricted to generating only lower and upper layer interneurons. Alternatively, these experiments may reveal that progenitors from these stages of MGE development have pluripotent characteristics with the capacity to switch their fates when placed into an older or younger environment. In the case of projection neurons, the classical transplantation experiments of McConnell and colleagues have defined a heirarchy of neuronal potency in heterochronic transplantations. Whereas early-born progenitors appear capable of populating both lower and upper cortical layers (McConnell, 1988; McConnell and Kaznowski, 1991), late-born projection neurons possess restricted potential and are able to contribute to only upper layers (Frantz and McConnell, 1996). Midstage progenitors appear to lie somewhere in between the two extremes with the capacity to contribute to upper layer fates but appear to have lost potential for neurons of the deepest layer 6 (Desai and McConnell, 2000). Those studies point to progressive changes in the intrinsic commitment of projection neuron progenitors over developmental time.

In contrast, a slightly different picture has emerged from the current experiments involving transplantation of MGE interneuron progenitors. Late-born progenitors (E15.5) were able to switch their fates, changing their normal upper layer destinations to lower layers when transplanted into a younger (E12.5) environment. The capacity of late-born interneurons to occupy lower layer positions normally reserved for early born-cells would suggest equivalent potency for interneuron populations born at these two different time points. In addition, it highlights the possibility that extrinsic, rather than intrinsic, properties are the dominant factors governing lower layer interneuron positioning. At the very least, certain intrinsic differences that are known to be present between early- and late-born cells appears to be inconsequential with respect to this issue. For instance, early-born interneurons are more dependent on Mash1 function, whereas lateborn neurons are more dependent on the function of $D l \times 1 / 2$ genes (Anderson et al., 1997b; Casarosa et al., 1999; Marin et al., 2000). In addition, late-born MGE cells are more sensitive to the branch-promoting and neurite-elongating effects of Slit protein compared with early-born neurons (Sang et al., 2002).

The importance of extrinsic cues for imparting layer information is also depicted by the distribution of transplanted E15.5 cells labeled with BrdU. In these experiments, BrdU was used to birthdate cells undergoing the final round of cell division, retaining the marker in transplanted neurons. By correlating BrdU adminis- tration in the host with the layer distribution of cells that have taken up the marker, it was possible to make inferences about the fate of neurons that had undergone the final cell division in the presence of BrdU. These BrdU data demonstrate that E15.5 neurons have switched to layer fates appropriate for the E12.5 environment, providing support to the principle that neuronal commitment to a particular layer is dependent on environmental cues received during the last round of cell division (McConnell and Kaznowski, 1991).

The results of transplanted late-born interneurons provide contrast to projection neuron experiments wherein late-born progenitors were found incompetent to respond to environmental cues involved in lower layer neuron positioning (Frantz and McConnell, 1996). Thus, it would appear that interneuron and projection progenitors, situated in different parts of the telencephalon but subsequently converging to form the upper cortical layers, have quite different intrinsic potentials to begin with. There are at least two possible reasons for this difference. Upper layer projection neurons are produced in vast numbers because of their generation at the end of proliferative (symmetric or asymmetric) cell cycles. Thus, at the time of upper layer neurogenesis, postmitotic projection neurons form the bulk of the nonproliferative fraction of cells present in the germinal zone (Takahashi et al., 1996). Therefore, the restriction of potency to upper layer neurons may be a shielding mechanism to avoid integration into the environments of lower and intermediate layers. In addition, their larger cell numbers ensures them greater success in colonizing upper layers, and restriction of potential is a small trade off. The second issue is the long distances that have to be covered by tangentially migrating interneurons. MGE cell migration may take up to $2 \mathrm{~d}$ to reach the extremity of their cortical destinations (Wichterle et al., 2001), and their lack of restriction may simply reflect greater flexibility that is required before their layer integration. This assertion is compatible with recent studies 
suggesting that interneurons may be naive until they reach the cortical VZ from which they seek layer address information (Nadarajah et al., 2002).

Conversely, experiments to test the potency of early-born cells were also performed by introducing early-born (E12.5) MGE cells into older (E15.5) hosts. The results revealed a shift in the destinations of transplanted cells toward the upper layers, indicating that early-born interneurons, normally destined for the lower layers, can be reprogrammed by the older environment to adopt upper layer fates. Interestingly, a large percentage of transplanted interneurons were located in bins 4 and 5, which are destinations not frequented by isochronic E15.5 transplants. Additionally, transplanted cells were also distributed in both the deep and superficial bins. These findings support the conclusion that early-born interneurons are pluripotent, and they occupied a wide range of cortical layers when transplanted into an older environment. It should be noted that this conclusion is derived from observing all $\beta$ gal + cells, regardless of whether they were postmitotic at the time of transplantation, or continued to divide once or multiple times in the host environment. To seek out the fates of neurons that have undergone the last cell division in the host environment, a BrdU pulse was delivered to the embryo after cell transplantation. The distribution of BrdU-labeled cells in the upper bins would suggest that these cells have responded appropriately to the layer-imparting cues of the older environment. It may be noted that other $\beta \mathrm{gal}+$ cells in the upper layers did not stain for BrdU (Fig. 8D). There are a possible number of reasons. These transplanted cells may also have completed their last cell division in the host environment and be subject to the upper layering cues. However, the transplanted cells may miss the BrdU label (available for a 2-3 hr time window) if they initiate S-phase after BrdU has been cleared from the host environment. Other $\beta$ gal + cells may have diluted out the BrdU marker with additional rounds of cell division. These results raise the question of whether neurons that had undergone the last cell division in the donor environment would also switch their fates. To address this, BrdU was delivered to the donor embryo at E12.5 before MGE dissection. After transplantation, these BrdU-labeled cells were found in the lower layers, suggesting that the MGE environment is capable of imparting layer-information cues to interneurons before their transplantation, at least for early-born neurons. This raises the question of whether late-born interneurons are also committed within the MGE environment to the upper cortical layers. Unfortunately, the present experimental tool is not informative on this point. Examination of the heterochronic transplants using E15.5 cells into E12.5 hosts showed hardly any cells in bins $7-10$, representing the upper layers (Fig. 5B). This would imply that the vast majority of transplanted cells have been respecified to occupy lower layers, including donor cells that may have undergone the last cell division in the MGE. Because very few labeled cells were found in the upper bins, it is not possible to test whether cells dividing in the MGE would only colonize the upper layers. A number of alternative interpretations may also be considered. The absence of upper layer labeling after transplantation into younger hosts may be attributable to failure of transplanted late-born neurons to survive long enough to participate in upper layer neurogenesis, even if they are irreversibly committed to the upper layers. Alternatively, E15.5 cells that divided last in the MGE may be specified for the upper layers but this specification is reversible, and, after transplantation into younger hosts, they are open to respecification into lower layer fates. If this latter point is true, it would suggest that late-born interneurons are less restricted in potential compared with early-born neurons.
This is not surprising given inherent differences between the two subpopulations. Late-born interneurons migrate through a deeper cortical route (Anderson et al., 2001) and are more dependent on $D l \times 1 / 2$ genes, whereas early-born interneurons are more sensitive to the effects of Mash1 (Casarosa et al., 1999; Marin et al., 2000). In addition, there is a population of late-born interneuron progenitors that appear to migrate into the cortex in which they subsequently proliferate (Anderson et al., 2001).

In summary, transplantation experiments allow the competing interests of intrinsic specification versus extrinsic reprogramming to be revealed. We show that cortical interneurons derived from the MGE possess layering information that allows them to be integrated into the neocortical environment in an inside-out sequence, despite their long and tortuous migratory journey. Interneuron progenitors born at a particular stage are not irreversibly committed to specific layers; instead, they appear open to respecification by the host environment and display pluripotent characteristics with respect to layer fates. Remarkably, despite their generation in a subcortical location, interneuron progenitors appear to share with projection neurons the same general principles of neuronal fating that results in the orderly assembly of six cortical layers.

\section{References}

Anderson S, Mione M, Yun K, Rubenstein JL (1999) Differential origins of neocortical projection and local circuit neurons: role of Dlx genes in neocortical interneuronogenesis. Cereb Cortex 9:646-654.

Anderson SA, Eisenstat DD, Shi L, Rubenstein JL (1997a) Interneuron migration from basal forebrain to neocortex: dependence on Dlx genes. Science 278:474-476.

Anderson SA, Qiu M, Bulfone A, Eisenstat DD, Meneses J, Pedersen R, Rubenstein JL (1997b) Mutations of the homeobox genes Dlx-1 and Dlx-2 disrupt the striatal subventricular zone and differentiation of late born striatal neurons. Neuron 19:27-37.

Anderson SA, Marin O, Horn C, Jennings K, Rubenstein JL (2001) Distinct cortical migrations from the medial and lateral ganglionic eminences. Development 128:353-363.

Anderson SA, Kaznowski CE, Horn C, Rubenstein JL, McConnell SK (2002) Distinct origins of neocortical projection neurons and interneurons in vivo. Cereb Cortex 12:702-709.

Angevine JB, Sidman RL (1961) Autoradiographic study of cell migration during histogenesis of the cerebral cortex in the mouse. Nature 192:766-768.

Bayer SA, Altman J (1991) Neocortical morphogenesis and histogenesis. New York: Raven.

Brustle O, Maskos U, McKay RD (1995) Host-guided migration allows targeted introduction of neurons into the embryonic brain. Neuron 15:1275-1285.

Campbell K, Olsson M, Bjorklund A (1995) Regional incorporation and site-specific differentiation of striatal precursors transplanted to the embryonic forebrain ventricle. Neuron 15:1259-1273.

Casarosa S, Fode C, Guillemot F (1999) Mash1 regulates neurogenesis in the ventral telencephalon. Development 126:525-534.

Chan C-H, Godinho LN, Thomaidou D, Tan S-S, Gulisano M, Parnavelas JG (2001) Emx1 is a marker for pyramidal neurons of the cerebral cortex. Cereb Cortex 11:1191-1198.

DeDiego I, Smith-Fernandez A, Fairen A (1994) Cortical cells that migrate beyond area boundaries: characterization of an early neuronal population in the lower intermediate zone of prenatal rats. Eur J Neurosci 6:983-997.

Desai AR, McConnell SK (2000) Progressive restriction in fate potential by neural progenitors during cerebral cortical development. Development 127:2863-2872.

Fagg GE, Foster AC (1983) Amino acid neurotransmitters and their pathways in the mammalian central nervous system. Neuroscience 4:701-719.

Fairen A, DeFelipe J, Regidor J (1984) Nonpyramidal neurons. In: Cerebral cortex (Jones EG, Peter AJ, eds), pp 201-254. New York: Plenum.

Fishell G (1995) Striatal precursors adopt cortical identities in response to local cues. Development 121:803-812. 
Franklin KBJ, Paxinos G (1997) The mouse brain in stereotaxic coordinates. San Diego: Academic.

Frantz GD, McConnell SK (1996) Restriction of late cerebral cortical progenitors to an upper-layer fate. Neuron 17:55-61.

Gilbert CD (1977) Laminar differences in receptive field properties of cells in cat primary visual cortex. J Physiol (Lond) 268:391-421.

Gilbert CD, Kelly JP (1975) The projections of cells in different layers of the cat's visual cortex. J Comp Neurol 163:81-105.

Gonchar Y, Burkhalter A (1997) Three distinct families of GABAergic neurons in rat visual cortex. Cereb Cortex 7:347-358.

Hendry SH, Jones EG (1981) Sizes and distributions of intrinsic neurons incorporating tritiated GABA in monkey sensory-motor cortex. J Neurosci 1:390-408.

Hendry SHC, Jones EG, Emson PC (1984) Morphology, distribution, and synaptic relations of somatostatin- and neuropeptide Y-immunoreactive neurons in rat and monkey neocortex. J Neurosci 4:2497-2517.

Hevner RF, Shi L, Justice N, Hsueh Y, Sheng M, Smiga S, Bulfone A, Goffinet AM, Campagnoni AT, Rubenstein JL (2001) Tbrl regulates differentiation of the preplate and layer 6. Neuron 29:353-366.

Jimenez D, Lopez-Mascaraque LM, Valverde F, De Carlos JA (2002) Tangential migration in neocortical development. Dev Biol 244:155-169.

Jones EG (1990) Modulatory events in the development and evolution of primate neocortex. In: Cerebral cortex (Jones EG, Peters A, eds), pp 311362. New York: Plenum.

Jones EG, Hendry SHC (1986) Co-localization of GABA and neuropeptides in neocortical neurons. Trends Neurosci 9:71-76.

Lavdas AA, Grigoriou M, Pachnis V, Parnavelas JG (1999) The medial ganglionic eminence gives rise to a population of early neurons in the developing cerebral cortex. J Neurosci 19:7881-7888.

Lin CS, Lu SM, Schmechel DE (1986) Glutamic acid decarboxylase and somatostatin immunoreactivities in rat visual cortex. J Comp Neurol 244:369-383.

Marin O, Rubenstein JL (2001) A long, remarkable journey: tangential migration in the telencephalon. Nat Rev Neurosci 2:780-790.

Marin O, Anderson SA, Rubenstein JL (2000) Origin and molecular specification of striatal interneurons. J Neurosci 20:6063-6076.

McConnell SK (1988) Fates of visual cortical neurons in the ferret after isochronic and heterochronic transplantation. J Neurosci 8:945-974.

McConnell SK, Kaznowski CE (1991) Cell cycle dependence of laminar determination in developing neocortex. Science 254:282-285.

Miller MW (1986) The migration and neurochemical differentiation of $\gamma$-aminobutyric acid (GABA)-immunoreactive neurons in rat visual cortex as demonstrated by a combined immunocytochemical-autoradiographic technique. Dev Brain Res 28:41-46.

Nadarajah B, Alifragis P, Wong RO, Parnavelas JG (2002) Ventricledirected migration in the developing cerebral cortex. Nat Neurosci $5: 218-224$

Naegele JR, Barnstable CJ (1989) Molecular determinants of GABAergic local-circuit neurons in the visual cortex. Trends Neurosci 12:28-34.

Olsson M, Bjerregaard K, Winkler C, Gates M, Bjorklund A, Campbell K
(1998) Incorporation of mouse neural progenitors transplanted into the rat embryonic forebrain is developmentally regulated and dependent on regional and adhesive properties. Eur J Neurosci 10:71-85.

Peduzzi JD (1988) Genesis of GABA-immunoreactive neurons in the ferret visual cortex. J Neurosci 8:920-931.

Polleux F, Whitford KL, Dijkhuizen PA, Vitalis T, Ghosh A (2002) Control of cortical interneuron migration by neurotrophins and PI3-kinase signaling. Development 129:3147-3160.

Rakic P (1974) Neurons in rhesus monkey visual cortex: systematic relation between time of origin and eventual disposition. Science 183:425-427.

Sang Q, Wu JY, Rao Y, Hsueh Y-P, Tan S-S (2002) Slit promotes branching and elongation of neurites of interneurons but not projection neurons from the developing telencephalon. Mol Cell Neurosci 21:250-265.

Schreyer DJ, Jones EG (1982) Growth and target finding by axons of the corticospinal tract in the prenatal and postnatal rat. Neuroscience 7:1837-1853.

Schwartz ML, Rakic P, Goldman-Rakic PS (1991) Early phenotype expression of cortical neurons: evidence that a subclass of migrating neurons have callosal axons. Proc Natl Acad Sci USA 88:1354-1358.

Streit P (1984) Glutamate and aspartate as transmitter candidates for systems of the cerebral cortex. In: Cerebral cortex (Jones EG, Peter AJ, eds), pp 119-143. New York: Plenum.

Sussel L, Marin O, Kimura S, Rubenstein JL (1999) Loss of Nkx2.1 homeobox gene function results in a ventral to dorsal molecular respecification within the basal telencephalon: evidence for a transformation of the pallidum into the striatum. Development 126:3359-3370.

Swanson LW (1998) Brain maps: structure of the rat brain. Amsterdam: Elsevier.

Takahashi T, Nowakowski RS, Caviness VS (1996) The leaving or Q fraction of the murine cerebral proliferative epithelium: a general model of neocortical neuronogenesis. J Neurosci 16:6183-6196.

Tamamaki N, Fujimori KE, Takauji R (1997) Origin and route of tangentially migrating neurons in the developing neocortical intermediate zone. J Neurosci 17:8313-8323.

Tan S-S, Breen SJ (1993) Radial mosaicism and tangential dispersion both contribute to mouse neocortex development. Nature 362:638-640.

Tan S-S, Kalloniatis M, Sturm K, Tam PPL, Reese BE, Faulkner-Jones BE (1998) Separate progenitors for radial and tangential cell dispersion during development of the cerebral neocortex. Neuron 21:295-304.

Van Eden CG, Mrzljak L, Voorn P, Uylings HBM (1989) Prenatal development of GABA-ergic neurons in the neocortex of the rat. J Comp Neurol 289:213-227.

Wichterle H, Garcia-Verdugo JM, Herrera DG, Alvarez-Buylla A (1999) Young neurons from medial ganglionic eminence disperse in adult and embryonic brain. Nat Neurosci 2:461-466.

Wichterle H, Turnbull DH, Nery S, Fishell G, Alvarez-Buylla A (2001) In utero fate mapping reveals distinct migratory pathways and fates of neurons born in the mammalian basal forebrain. Development 128:37593771. 